\title{
CLUSTER COMPUTING OF HIGH FREQUENCY ELECTROMAGNETIC FIELD PROPAGATION
}

\author{
Wojciech Walendziuk ${ }^{1)}$, Jaroslaw Forenc ${ }^{2)}$, Andrzej Jordan ${ }^{3)}$ \\ ${ }^{1)}$ Bialystok Technical University, Wiejska 45D Street, 15-351 Bialystok, POLAND \\ e-mail: jarekf@pb.bialystok.pl,we.pb.bialystok.pl \\ ${ }^{2)}$ Bialystok Technical University, Wiejska 45D Street, 15-351 Bialystok, POLAND \\ e-mail: walenw@pb.bialystok.pl,we.pb.bialystok.pl \\ 3) Polish-Japanese Institute of Information Technology, Koszykowa 86 Street, 02-008 Warsaw, POLAND \\ e-mail: jordana@pjwstk.edu.pl,www.pjwstk.edu.pl
}

\begin{abstract}
The paper presents parallel implementations of the FDTD (Finite Differences Time Domain) method in cluster systems of PC's. Those two- and three-dimensional algorithms are based on the stripes decomposition of the analyzed area. What is more, the communication among particular computation nodes building the cluster system is discussed. On the basis of the obtained results, the analysis of the efficiency of the presented algorithms was done.
\end{abstract}

Keywords: FDTD method, electromagnetic field, cluster computing.

\section{INTRODUCTION}

In the numerical analysis of the high frequency electromagnetic fields the FDTD (The FiniteDifference Time-Domain) method is often used $[1,2]$. This method is relatively simple in numerical implementation and can be used in analysis of systems of very complicated geometry in homogeneous and heterogeneous mediums. The FDTD method is based on Maxwell time dependent equations (1) which are directly integrated in time and space domains.

$$
\nabla \times \vec{E}=-\mu \frac{\partial \vec{H}}{\partial t} \quad \nabla \times \vec{H}=\sigma \vec{E}+\varepsilon \frac{\partial \vec{E}}{\partial t}
$$

where: $\vec{E}$ - vector of the electric field intensity $[\mathrm{V} / \mathrm{m}], \vec{H}$ - vector of the magnetic field intensity $[\mathrm{A} / \mathrm{m}], \mu$ - magnetic permeability $[\mathrm{H} / \mathrm{m}], \sigma$ - electric conductivity $[\mathrm{S} / \mathrm{m}], \varepsilon$ - electric permittivity $[\mathrm{F} / \mathrm{m}]$.

The numerical realization of computations is possible due to the use of Yee algorithm [3]. Making a decomposition of the equation (1) using the Cartesian coordinates into particular electromagnetic field components, we obtain six scalar equations. Then, these equations are transformed to a differential form according to the Yee cell construction (Fig.1). In the nodes of each such a cell, a)
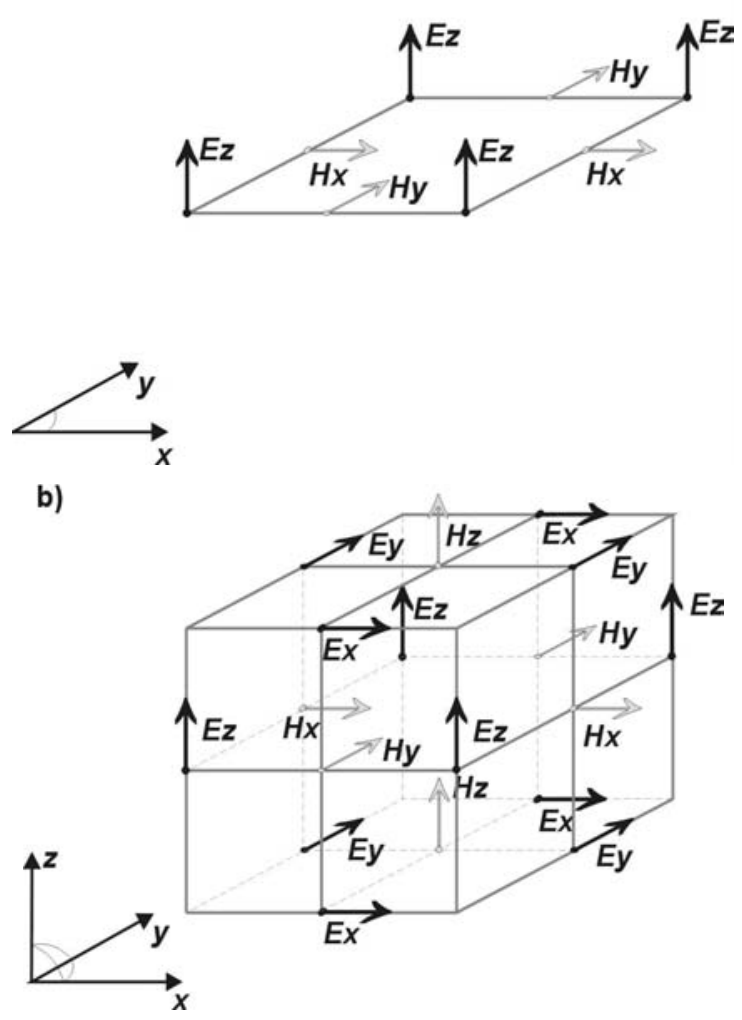

Fig.1 - Proper location of the components of the electric and magnetic field intensity vectors in an elementary Yee cell for a two-dimensional TM

(Transverse Magnetic) case (a) and a threedimensional case (b). 
particular components of electric field intensity vectors $\vec{E}\left(E_{x}, E_{y}, E_{z}\right)$ and magnetic filed intensity $\vec{H}\left(H_{x}, H_{y}, H_{z}\right)$ are placed. In such a case, the analyzed area is filled up with these Yee cells, and each node of the Yee cells stands for a node of the finite difference grid in numerical algorithms.

On the surfaces edging the examined area absorbing boundary conditions should be assumed as Mur's [4], Mei's [5] or PML [6].

The use of the FDTD method in the electromagnetic fields of high frequency analysis in complicated structures, especially when high accuracy of computations is needed, requires using computation systems of great computational efficiency and of high capacity of the operational memory. The solution of this problem may be the use of parallel computers or cluster systems.

In the paper, a parallel implementation of the FDTD method algorithm in cluster systems of PC's will be presented in both versions: a two- and a three-dimensional one.

\section{THE PARALLEL ALGORITHM OF THE FDTD METHOD}

The parallel implementation of the FDTD algorithms based on the division of the analyzed area into sub-areas in which the computations are conducted in parallel through particular nodes. In such a case, it is necessary to use the Master-Slave topology. Then, the Master computer initiates the computation process and the Slave computers take the role of independent computation nodes working with the same computational algorithm. The general course of the computation process may be presented as follows:

1. Master: sends data which is necessary to initiate computations to the computing nodes.

2. Slave: receives data initiating computations.

3. Slave: memorizes data needed to compute the components of the Mur's absorbing boundary conditions of the first order.

4. Slave: computes the electric intensity components $\vec{E}$.

5. Slave: computes components of the Mur's absorbing boundary conditions.

6. Slave: data exchange between neighboring computation nodes - $\vec{E}$ components.

7. Slave: computes the magnetic intensity components - $\vec{H}$ components.

8. Slave: data exchange between neighboring computation nodes - $\vec{H}$ components.

9. Slave: sends selected results of computations to the Master processor.

10.Master: records the results of computations on the local hard disk.
In the FDTD method the computations are stopped after a particular number of algorithm steps, established in advance. Thus, the $3 \div 10$ stages are repeated many times. It must be mentioned that during the process of computation initiation the data concerning the size of the analyzed space, the material parameters of analyzed space and places of divisions into sub-areas are sent to the computation nodes.

\section{A TWO-DIMENSIONAL ALGORITHM OF THE FDTD TM (TRANSVERSE MAGNETIC CASE) METHOD}

The computations were carried out with the use of a homogenous cluster system (Fig.2) which consisted of seven PC's (six computation nodes and one control unit - Master processor). Each node was equipped with an Intel Pentium II $866 \mathrm{MHz}$ processor, $128 \mathrm{MB}$ RAM and a 40GB hard disk. The computers were working under control of the Microsoft Windows ME operational system, yet the communication among processors was realized with the use of an implementation of the MPI (Message Passing Interface) standard - WMPI v1.2 [7]. The computers used for computations were connected via the Ethernet $10 \mathrm{Mb} / \mathrm{s}$ (the real achieved bandwidth was about $2 \mathrm{Mb} / \mathrm{s}$ ). Such a connection significantly aggravates the efficiency of the work of parallel algorithm. It is worth stressing that using such a cluster of PCs was determined by the fact that the machines are normally used for everyday didactic purposes.

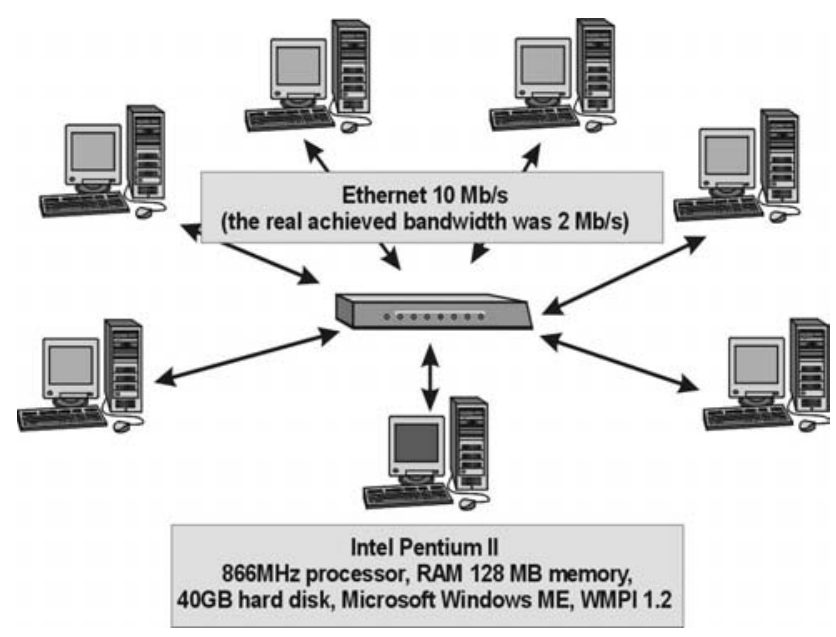

Fig.2 - A homogenous cluster system.

In a parallel algorithm of the two-dimensional FDTD method the space domain was divided into „stripes" $[8,9]$. Such a decomposition is based on the division of the examined area into smaller parts in which communication takes place only on the parallel edges of the division. Particular computation nodes conduct parallel computations, and then 
exchange data needed to initiate the following

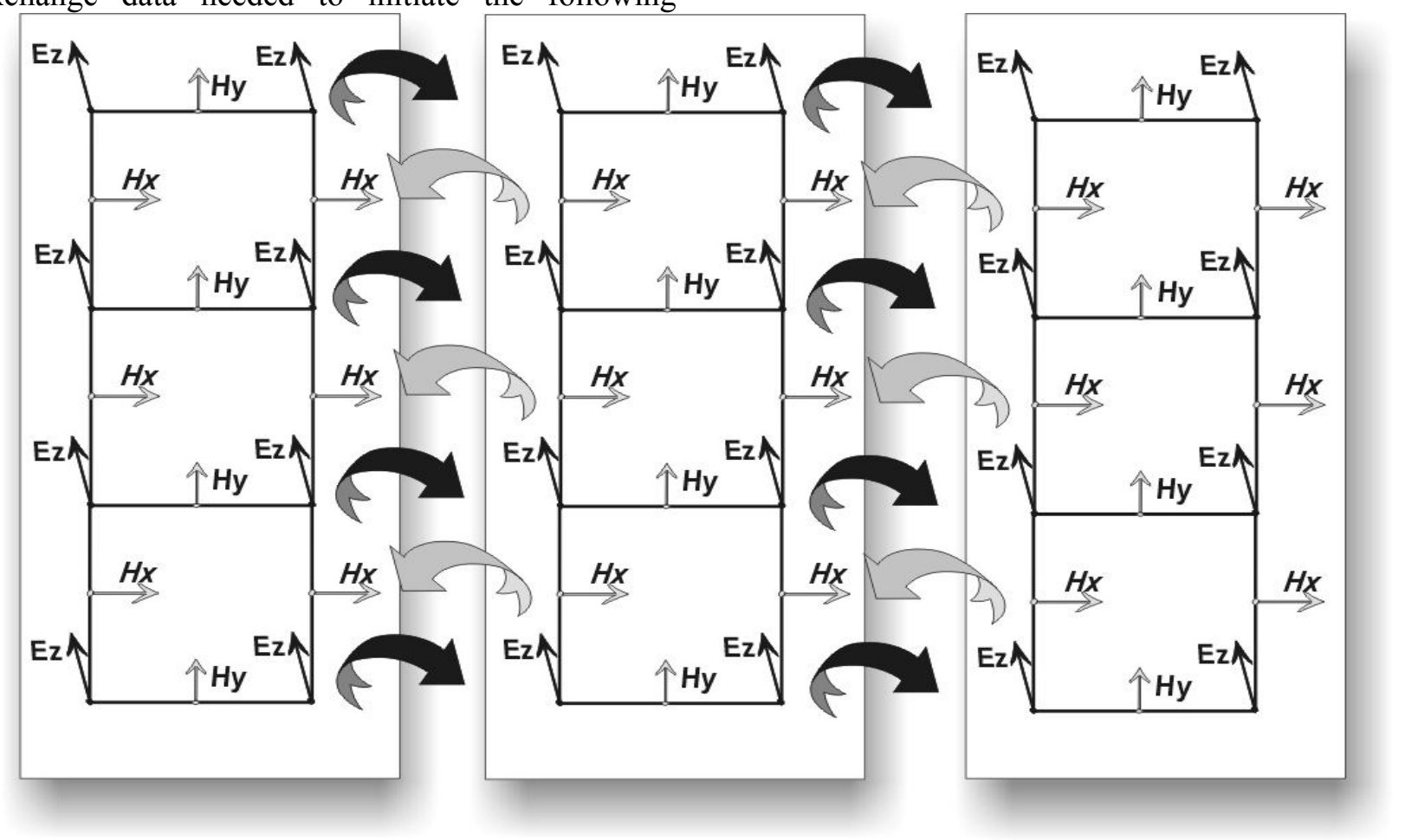

Computation Node 1

Computation Node 2

Computation Node 3

Fig.3 - The "stripe" decomposition with the communication data exchange.

computation step. As it can be observable in Fig.3, only $H_{y}$ (the grey arrows) and $E_{z}$ (the black arrows) components data exchange occurs. The sub-areas computed in particular nodes are of the same size. Therefore, the quantity of the data exchanged among nodes during their communication is equal.

During a parallel algorithm efficiency examination it was observable that the speedup, defined as the coefficient of the total computation time of the sequential implementation measured on a single machine, to the total computation time of a parallel program run on $n$ machines, rises with the increase of the number of the finite differences nodes. With the grid density of $1500 \times 1500$ Yee cells, the achieved speedup was 4.8 for six computation nodes and one control node (Fig.4).

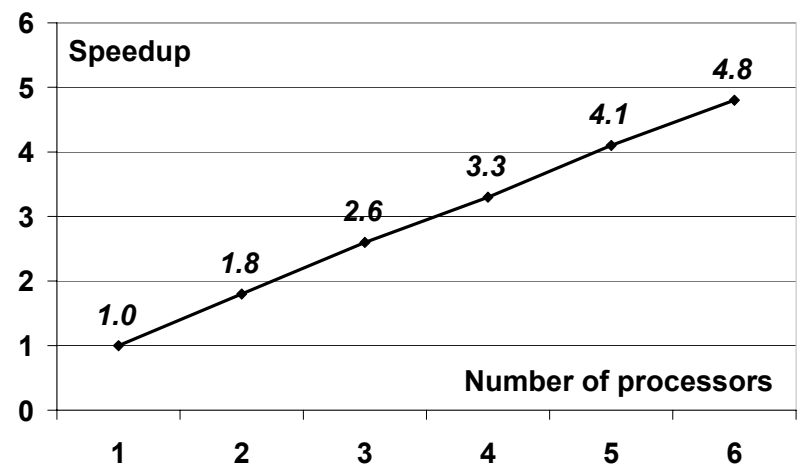

Fig.4 - The achieved speedup in the two-dimensional algorithm of the FDTD TM method.

\section{A THREE-DIMENSIONAL ALGORITHM OF THE FDTD METHOD IN A HETEROGENEOUS CLUSTER SYSTEM}

The next phase of the research was the creation and examination of a three-dimensional parallel implementation of the FDTD method algorithm. The computations were carried out with the use of heterogeneous cluster system (Fig.5) consisting of seven PCs (six computations nodes and one control unit), working under the Microsoft Windows 2000 PL operational system.

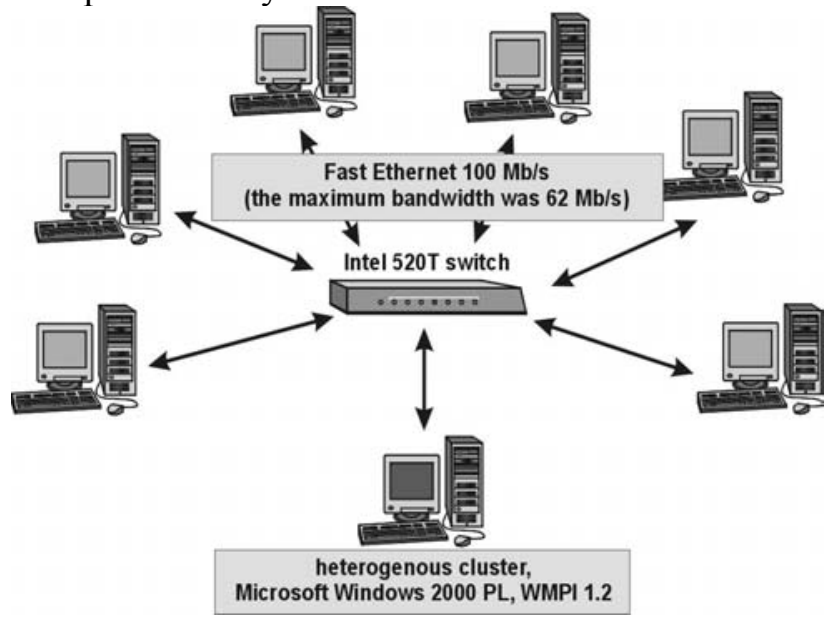

Fig.5 - A heterogeneous cluster system.

The choice of the operational system was determined mainly by the computers assignment - 
they are not only used for computations, but also for everyday work. The computers were connected with the use of Fast Ethernet network $(100 \mathrm{Mb} / \mathrm{s})$, by the Intel 520T switch. Such a cluster system is fast enough to conduct numerical computations. The maximum achieved bandwidth of the data transmission in this network was about $62 \mathrm{Mb} / \mathrm{s}$. Similarly, as in the previous, two-dimensional case, the WMPI v1.2 communication library was used.

In the parallel three-dimensional implementation of the FDTD method algorithm, components of the magnetic field intensity vector $H_{y}$ and $H_{z}$ are sent to the left "neighbor" and received from the right "neighbor" [10]. During the next time step activities of the algorithm, the electric field intensity components $E_{z}$ and $E_{y}$, which are already calculated, are sent to the right "neighbor" and received from the left "neighbor" (Fig.6). The remaining components $E_{x}$ and $H_{x}$, do not have to be sent, according to the construction of the K.Yee algorithm, used in the FDTD method.

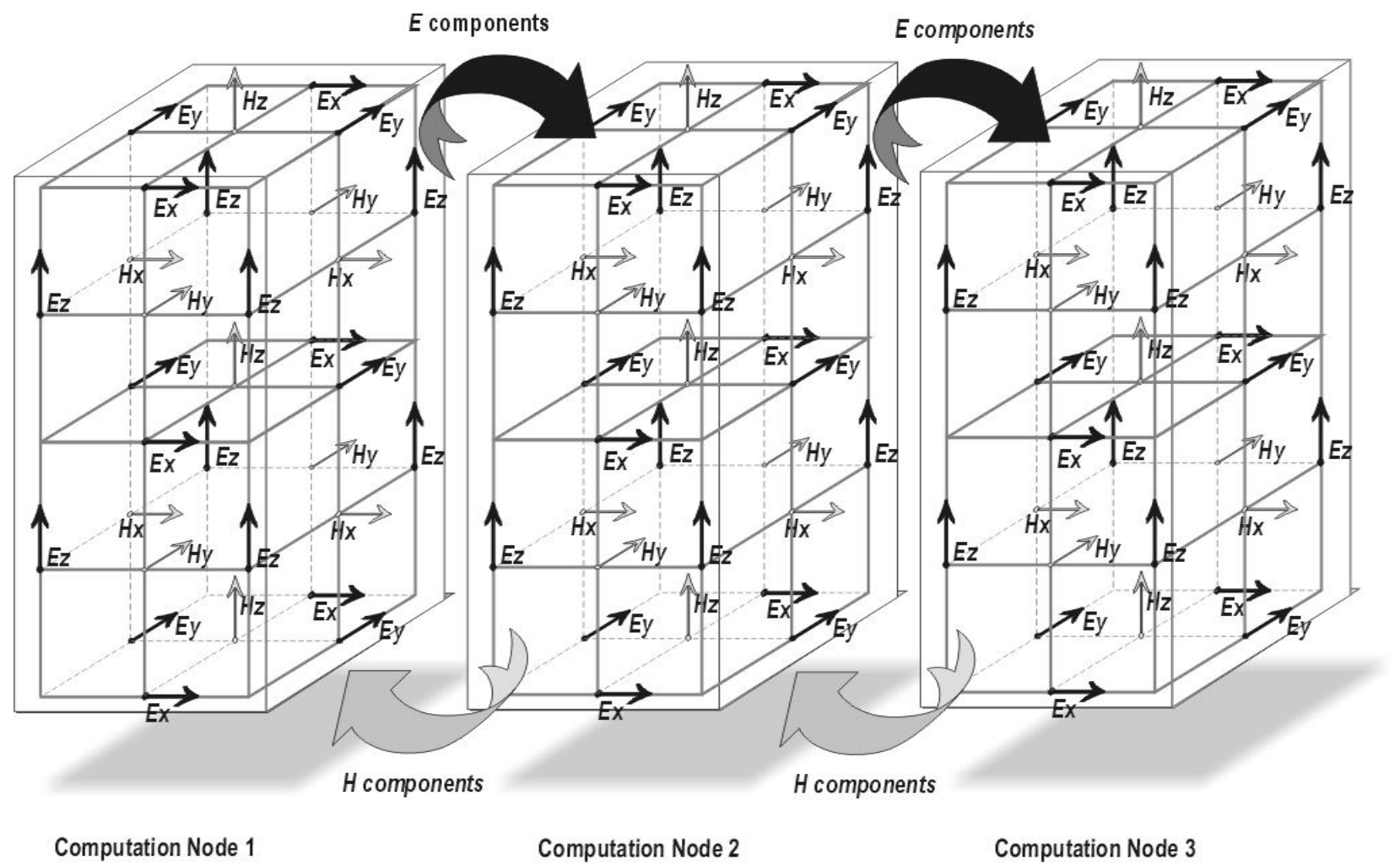

Fig.6 - The "slice" decomposition with the data exchange.

The presented algorithm was tested on the system with the grid density of $60 \times 60 \times 60$ Yee cells (thus the total number of the Yee cells was 216000).

Fig.7 shown the achieved speedup. At the six computation nodes it accounted for about 5.3.

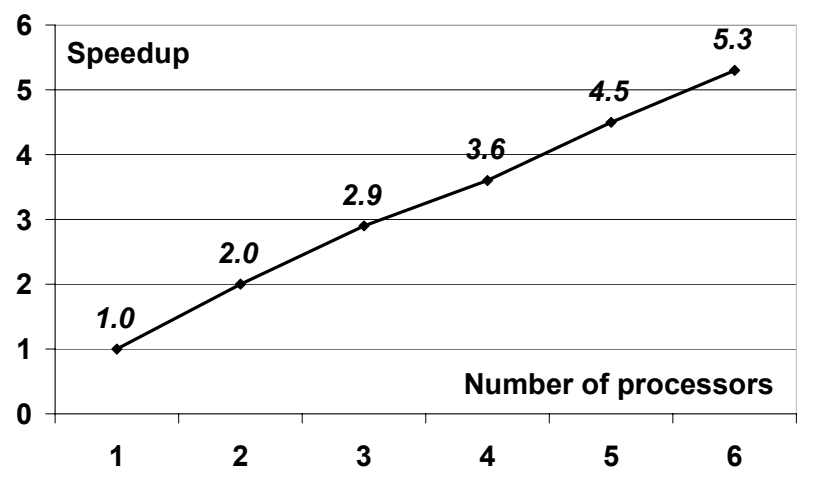

Fig.7 - The achieved speedup in the three-dimensional algorithm of the FDTD method.

\section{CONCLUSIONS}

In the article, two examples of the parallel implementation of the FDTD method in cluster systems are presented. The two-dimensional algorithm was implemented in a homogenous cluster system, and the three-dimensional algorithm - in a heterogeneous system. The use of cluster computing in the high frequency electromagnetic fields analysis brings an opportunity to accelerate significantly the time of computations. Another important advantage is the possibility of enlarging the examined area or the accuracy of computations, even till the size limited by the capacity of the operational memory of all the nodes in the cluster. During the parallel algorithm efficiency examination, satisfying results of the speedup were obtained. Moreover, it was observed that the speedup was increasing together with the enlargement of the examined area in 
particular nodes. In both algorithms presented here, the analyzed area was divided into equal parts. Therefore, each computational node received equal area of computations. In the case of the heterogeneous cluster systems consisting of computational nodes of variable parameters, it would be appropriate to use the division of the analyzed area adjusted to the efficiency of a particular nodes. Such a division should minimize possible waiting times of ,faster" nodes for the „slower" nodes to complete their work.

\section{Acknowledgement:}

The work has been sponsored by KBN grant 4T11C 00722 and an internal PJIIT research grant.

\section{REFERENCES}

[1] A. Taflove, S.C. Hagness. Computational Electrodynamics, The Finite - Difference Time Domain Method. Artech House, Inc. Boston, 2000.

[2] K.S. Kunz, R.J. Luebbers. The Finite Difference Time Domain Method for Electromagnetics. Boca Raton, CRC Press, Inc., 1993.

[3] K.S. Yee. Numerical Solution of Initial Boundary Value Problems Involving Maxwell's Equations in Isotropic Media, IEEE Transactions on Antennas and Propagation AP-14 (3) (1966). p. $302-307$

[4] G. Mur. Absorbing Boundary Conditions for the Finite Difference Approximation of the TimeDomain Electromagnetic-Field Equations, IEEE Transactions on Electromagnetic Compatibility EMC-23 (4) (1981).p.377-382

[5] K.K. Mei, J. Fang. Superabsorption - A Method to Improve Absorbing Boundary Conditions. IEEE Transactions on Antennas and Propagation Vol. 40 (9) (1992). p.1001-1010

[6] J.P. Berenger. A Perfectly Matched Layer for the Absorption of Electromagnetic Waves. Journal of Computational Physics Vol. 114 (1) (1994). p.185-200

[7] J. Marinho, J.G. Silva. WMPI - Message Passing Interface for Win32 Clusters. "Proceedings of 5th European PVM/MPI User's Group Meeting", 1998, pp.113-129.

[8] W. Walendziuk, J. Forenc. Decomposition of the computation area in the parallel implementation of the FDTD algorithm. "International Symposium on Parallel and Distributed Computing (ISPDC'2002)", Iaşi, Romania, 17-20 July 2002, pp. 180-192.

[9] W. Walendziuk. SAR and thermal computations with the use of parallel FDTD algorithm. "International Conference on Parallel Computing in Electrical Engineering
(PARELEC'2002)", Warsaw, Poland, 22-25 September 2002, pp.282-287.

[10]J. Forenc, A. Skorek. Analysis of High Frequency Electromagnetic Wave Propagation Using Parallel MIMD Computer and Cluster System, "International Conference on Parallel Computing in Electrical Engineering (PARELEC'2000)", Trois-Rivères, Canada, 2730 August 2000, pp.176-180.

Wojciech Walendziuk was Born in Elk, Poland, in 1972. He studied electrical engineering at the Bialystok Technical University where he recived the M.Sc. in 1998. In the same year, he has been employed at the university as a Researchers and

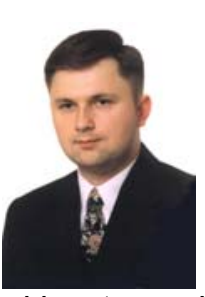
Teaching Assistant. He is currently working toward the Ph.D. degree at the same university. The main areas of his research are parallel algorithms in electrical engineering, high frequency electromagnetic field propagation and biological effects of electromagnetic fields radiation.

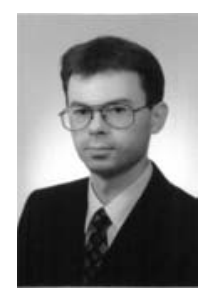

Jaroslaw Forenc was born in Kolno, Poland, on May 26, 1972. He received the M.Sc. degree in information technology from the Bialystok Technical University in 1997. In the same year, he has been employed at the Białystok Technical University, Faculty of Electrical Engineering as a Teaching and Research assistant. $\mathrm{He}$ is currently working toward the Ph.D. degree at the same university. His research interests include parallel computations in electrical engineering, and parallel analysis of transient states in physical systems.

Andrzej Jordan received the Ph.D. degree and habilitation degree in the electrical engineering from the Technical University of Lodz, Poland, in 1970 and 1979, respectively. He is currently the Professor with the Faculty of Electrical Engineering of

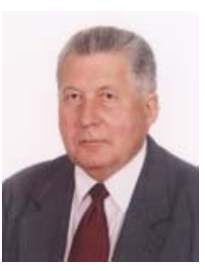
the Bialystok Technical University (BTU). From 1973 he is the head of the chair of Theoretical Electrotechnics and Metrology in the BTU. His main research interests include computational electromagnetism, parallel computing and the use of evolutionary strategies for physical problems solving and their optimization. 